\title{
THE ROLE OF WIRELESS NETWORK IN REMOTE MONITORING OF ELECTROMECHANICAL EQUIPMENT
}

\author{
Liang Guo ${ }^{1}$, Viet Hung Luu' ${ }^{2}$, Shahreen Kasim ${ }^{3}$ \\ ${ }^{1}$ Department of Mathematics and Information Engineering,Dongchang College of Liaocheng University \\ Shandong Liaocheng 252000, China \\ ${ }^{2}$ Maritime College No. 1, Haiphong, Vietnam \\ Faculty of Computer Science and Information Technology, Universiti Tun Hussein Onn Malaysia \\ Email: liangguo201033@126.com
}

\begin{abstract}
To study the remote monitoring electromechanical equipment of wireless network, the wireless vibration sensor monitoring system is designed for rotating mechanical equipment. Firstly, for the overall design of the system, problems to be considered in the design process are proposed, and the system structure is improved in this research. Secondly, the wireless vibration monitoring node and the wireless vibration base station are modularized, and the devices details are given. Thirdly, the communication protocol of wireless network is designed, the topology of wireless network is improved, and the packet format is standardized. Finally, the data transmission performance of the designed monitoring system is tested and compared with that of YD-82 piezoelectric acceleration sensor. The results show that after 10 data transmission experiments, the data loss rate is lower than $0.2 \%$. Furthermore, the frequency and amplitude of the main spectral lines of YD-82 sensor are close to that of the monitoring system designed in this study, whereas, the spectral line amplitude of the monitoring system is lower than that of YD-82 sensor. Therefore, the wireless network monitoring system designed in this study can meet the vibration monitoring requirements of electromechanical equipment.
\end{abstract}

Keywords: Electromechanical equipment, Remote monitoring, Monitoring nodes, Monitoring Base Station, The Wireless Network.

\section{Introduction}

With the improvement of people's living standards, the requirements for mechanical and electrical equipment are becoming higher and higher. Vehicles, household appliances, and work appliances have become indispensable mechanical and electrical products in daily life [1]. In recent years, with the development of science and technology, the competition among various industries has promoted the industrial manufacturing to complete the transformation and upgrading of large-scale mechanical and electrical equipment and automation, which has made outstanding contributions to reducing corporate costs and improving economic benefits [2]. At the same time, higher requirements are observed for the performance of mechanical and electrical equipment. Failure of mechanical and electrical equipment will seriously affect the whole production process and cause significant economic losses [3]. To reduce the impact caused by mechanical and electrical equipment failure, researchers ensured focus on the monitoring of mechanical and electrical equipment and invented a series of mechanical and electrical equipment monitoring systems [4-6].

The traditional monitoring system has fewer inspection points and shorter data transmission distance, and most of the data collection and processing are conducted by wire method. Although it can realize real-time data transmission, it has high laying cost, complicated wiring, and certain defects in equipment maintenance [7-8]. With the development of network communication technology, aiming at the solution-oriented problems of remote monitoring objects, multiple monitoring types, and scattered monitoring points, the use of wireless sensor network for remote monitoring of electromechanical equipment has become an inevitable trend [9-10]. The monitoring system of wireless sensor network can obtain the vibration signal of the equipment and transmit the wireless data through the network of sensor nodes, which can cover the defects and deficiencies of the wired monitoring network [11].

However, at present, the application of wireless sensor network to the monitoring of rotating mechanical equipment is not mature, and it is often 
applied to the signal monitoring of low vibration system, with large data volume and low transmission rate [12-13].

Based on mentioned deficiencies presented study propose the wireless vibration sensor monitoring system. designed for rotating mechanical equipment. The wireless vibration monitoring node and the wireless vibration base station are modularized to improve the topology of the wireless network, and the packet format is standardized. It is expected to meet the vibration monitoring requirements of electromechanical equipment.

\section{Methods}

In the design of wireless remote monitoring system for rotating machinery equipment, primarily includes wireless network monitoring node and base station. Wireless network monitoring nodes include control center, data collection and storage, radio frequency transmission, and power supply. While, nodes collect and convert the information of rotating mechanical equipment into digital format and transmit it to the base station through wireless network. The base station of wireless network includes control center, data storage, radio frequency transmission, Ethernet communication, and power supply. The role of the base station is to integrate and classify the node information added to the wireless network, transmit data through Ethernet to the top computer, and carry out data processing, display, storage, and other operations.

\subsection{Overall system design}

In the process of system design, the following issues need to be considered through the detection and analysis of equipment conditions and environment:
First, the size of wireless network nodes. The wireless network nodes need to be connected to the rotating mechanical equipment through bolts, and the rotating mechanical equipment has a small spatial structure. If the nodes are too large, it is difficult to adapt the complex installation environment. Therefore, the volume of wireless network nodes should be kept as small as possible.

Second, the energy consumption and cost of wireless network nodes. In the system design, wireless transmission is adopted to reduce the cost of equipment, and the monitoring node in such situation uses battery power. To extend the service life of monitoring nodes, the designed wireless network nodes should not only meet the requirements of node performance, but also choose devices with low energy consumption to reduce the cost.

Third, wireless network communication methods. In the monitoring system, data communication is conducted between the monitoring node and the base station through wireless transmission. Wireless transmission methods mainly include $\mathrm{WiFi}$, Bluetooth, radio frequency, and Zigbee, etc., and their respective characteristics are shown in Table 1.

Fourth, the communication between the wireless network base station and the host computer. The base station of wireless network is composed of the data gathered from the nodes of wireless network. It does not need to monitor the rotating mechanical equipment, so the layout requirements of the base station are not high. The communication between the wireless network base station and the host computer can be carried out through Ethernet.

Through the correlation analysis of the above system, the system structure includes wireless network monitoring nodes, wireless network base station, and wireless network upper computer monitoring software, as shown in figure 1.

Table 1. Characteristics of wireless transmission methods

\begin{tabular}{|c|c|c|c|c|}
\hline & WiFi & Bluetooth & Radio frequency & Zigbee \\
\hline Covering distance over m & $>100$ & $10-100$ & $>1000$ & $>75$ \\
\hline Transmission rate / bps & $1-11 \mathrm{M}$ & $<1 \mathrm{M}$ & $>10 \mathrm{k}$ & $250 \mathrm{k}-1 \mathrm{M}$ \\
\hline Frequency band / Hz & $2.4 \mathrm{G}$ & $2.4 \mathrm{G}$ & $100-1000 \mathrm{M}$ & $868 \mathrm{M}-2.4 \mathrm{G}$ \\
\hline Energy consumption and cost & Low & Low & Low & Low \\
\hline Network usage fee & None & None & None & None \\
\hline Integration and reliability & High & High & High & High \\
\hline Application range & Family, office & Family, office & Industry & Industry \\
\hline
\end{tabular}




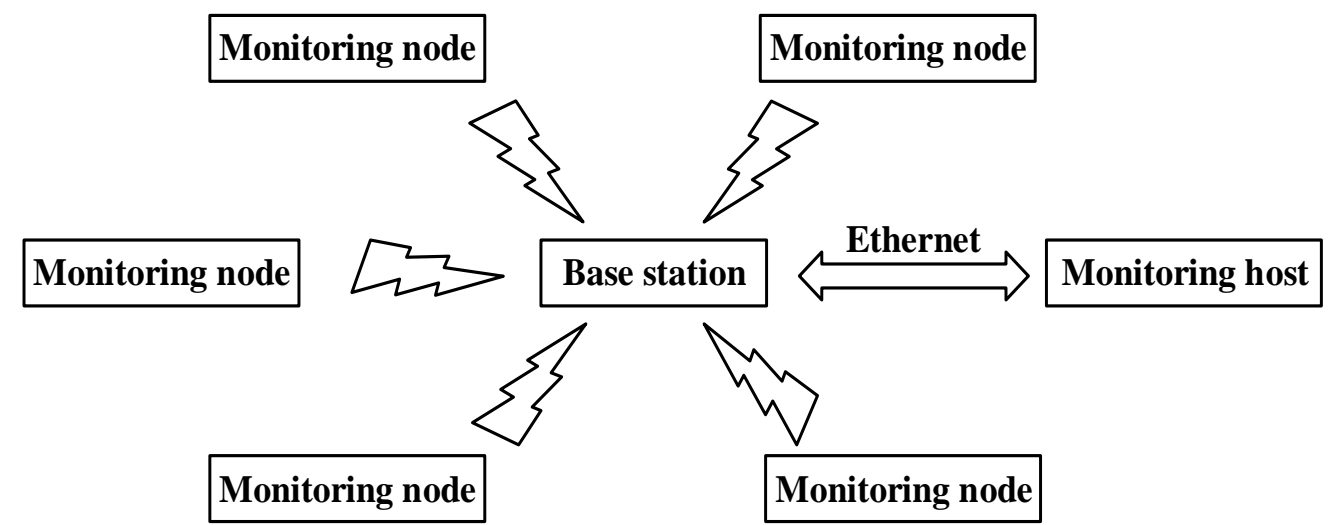

Figure 1: Structure of a wireless network remote monitoring system

The monitoring node on the rotating mechanical equipment can obtain the operation information of the equipment and transmit it to the base station by radio frequency. The base station transmits the information to the monitoring host through Ethernet. The host computer act as the data center of the whole monitoring system. The base station receives the control instructions of the host computer and transmits them to the target monitoring node through radio frequency. Users can observe and analyze the monitoring data and understand the equipment status through the monitoring host.
Wireless vibration monitoring network is a star network structure, including a network center and several network nodes. The network center is the base station of the wireless network, and the network nodes are the monitoring nodes of the wireless network. These constitute the first-level wireless star network structure. The secondary wireless star network structure is wired, with the network center as the monitoring host and the network node as the wireless network base station. The two-stage star network structure transfers data to the top machine by wireless and wired means, as shown in figure 2 .

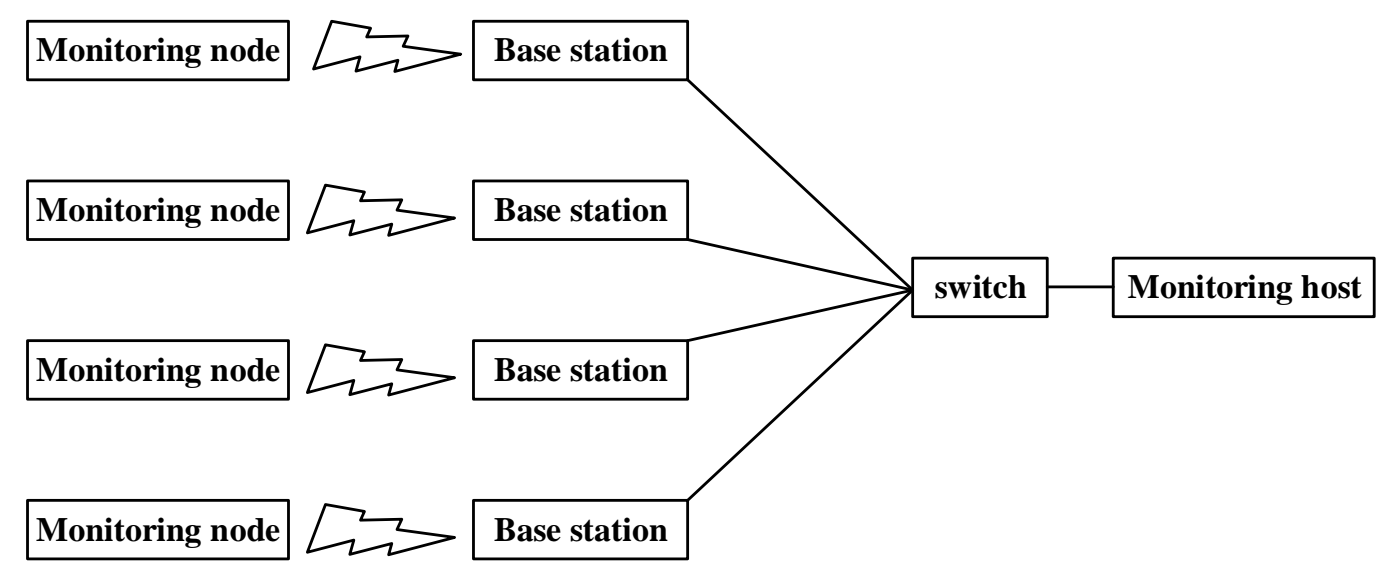

Figure 2: Structure of the improved wireless network remote monitoring system

\subsection{Design of wireless vibration monitoring node}

The precision and sampling rate of rotating mechanical equipment are required to be high, and a large amount of data will be generated during monitoring. To solve the power consumption problem caused by a large amount of data, the monitoring node is required to have high computing capacity, low energy consumption, and high storage capacity.

The design scheme of wireless network monitoring node is shown in figure 3. Wireless network monitoring nodes include control center, data acquisition and storage, radio frequency transmission, and power supply, which are designed with modularization to increase or decrease different functional modules. 


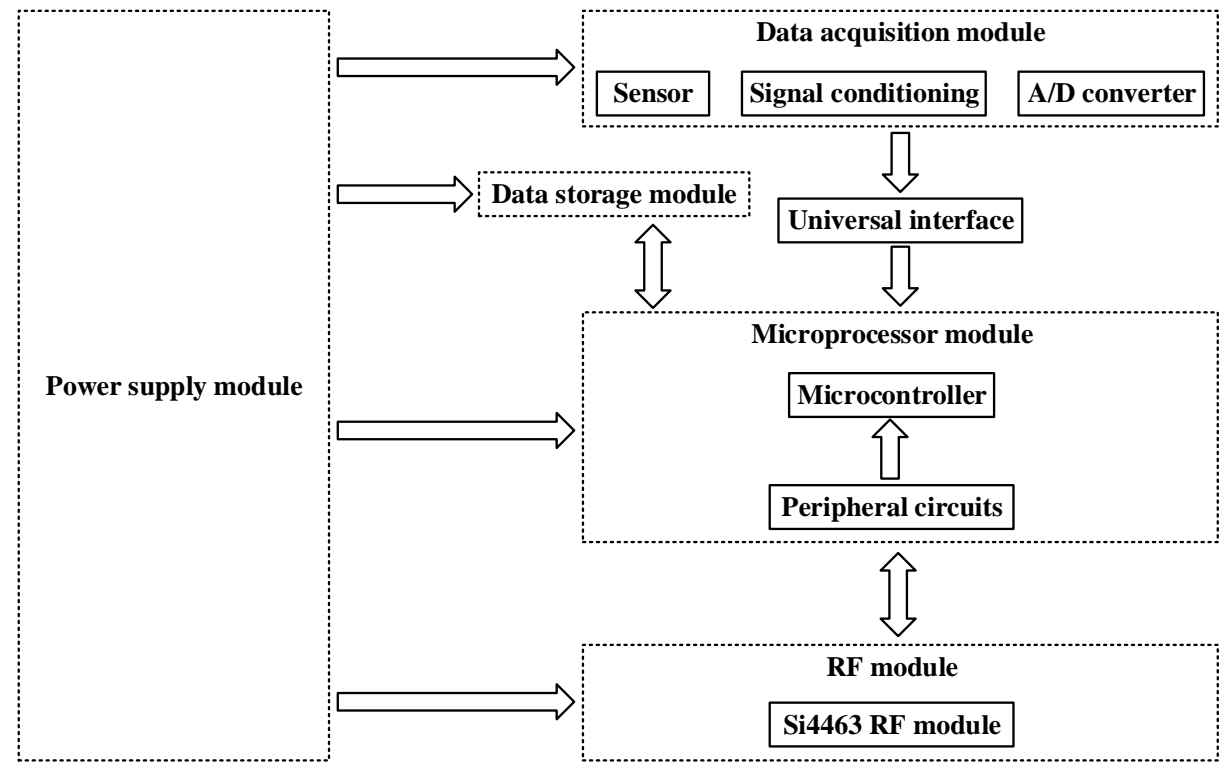

Figure 3: Design of wireless network monitoring nodes

As the key part of the monitoring node, the processor determines the computing power and energy consumption cost of the monitoring node. In the system designed in this study, the 32-bit microprocessor STM32F405RG with ARM Cortex-M4 kernel is adopted. When it runs at the maximum frequency of $168 \mathrm{MHz}$, the operation efficiency is 210 DMIPS, which meets the data processing requirements of the monitoring node. The STM32F405RG also has many external device interfaces and ultra-low energy consumption.

The radio frequency module is responsible for the operation of wireless communication protocol, node networking, and data receiving. In the system designed in this study, Si4463 radio frequency chip is adopted, with excellent performance and low energy consumption. The frequency band is between 119 and $1050 \mathrm{MHz}$, which can support a variety of modulation modes. The transmission rate is $1 \mathrm{Mbps}$, the reception sensitivity is $-126 \mathrm{dBm}$, and the maximum output power is $+20 \mathrm{dBm}$.

The circuit of data acquisition module includes two parts, that is, sensor and signal conditioning conversion. The sensor measures the vibration parameters of the equipment, so the sensor should meet the requirements of small size, low energy consumption, simple circuit, and so on. In this study, the MEMS vibration acceleration sensor ADXL22035 is adopted, with high precision and low energy consumption. It is a uniaxial accelerometer, and the main indicators are shown in table 2.

Table 2. Technical specifications of ADXL22035

\begin{tabular}{|c|c|c|c|}
\hline Single power supply & $3.0-6.0 \mathrm{~V}$ & Resolution & $1 \mathrm{mg}(60 \mathrm{~Hz})$ \\
\hline Acceleration range & $\pm 18 \mathrm{~g}$ & Sensitivity & $100 \mathrm{mV} / \mathrm{g}$ \\
\hline $\begin{array}{c}\text { Measurement } \\
\text { bandwidth }\end{array}$ & $\begin{array}{c}0.5-2.5 \mathrm{kHz}, \\
\text { dynamically adjustable }\end{array}$ & Energy consumption & $700 \mu \mathrm{A}$ \\
\hline
\end{tabular}

The microprocessor can only recognize and process digital signals. Therefore, when collecting analog signals, it first adjusts the analog signals and converts them into digital signals. In this conversion process, A/D converter is used. Since the signal range of $A / D$ converter is different from the system requirements, the output signal of the level conversion sensor needs to be adjusted to the signal range of the $A / D$ converter. In this study, the A/D converter model is AD7685, while sampling rate is kept $250 \mathrm{kSPS}$ with 16-bit conversion accuracy, the
SNR is $93.5 \mathrm{~dB}$, and the single power supply remain between 2.3-5.5 V.

Moreover, the transmission rate of wireless network is difficult to achieve the real-time transmission requirements of massive high-speed signals, so the rotating mechanical vibration monitoring nodes need large data storage and memory with high access speed. A comparison of several common memories is shown in table 3 . It can be concluded that NOR FLASH is suitable for data storage. 
Table 3. Properties of common memories devices

\begin{tabular}{|c|c|c|c|c|c|c|}
\hline & SRAM & DRAM & MRAM & EEPROM & $\begin{array}{c}\text { NOR } \\
\text { FLASH }\end{array}$ & NAND FLASH \\
\hline Capacity & $\begin{array}{c}256 \mathrm{~KB}-16 \\
\mathrm{MB}\end{array}$ & $16 \mathrm{MB}-4 \mathrm{~GB}$ & $\begin{array}{c}128 \mathrm{~KB}-16 \\
\mathrm{MB}\end{array}$ & $1 \mathrm{~KB}-1 \mathrm{MB}$ & $\begin{array}{c}256 \mathrm{~KB}-64 \\
\mathrm{MB}\end{array}$ & 128 MB-4 GB \\
\hline Erasing speed & Infinite & Infinite & Infinite & $\begin{array}{c}100,000 \\
\text { times }\end{array}$ & $\begin{array}{c}100,000 \\
\text { times }\end{array}$ & $\begin{array}{c}1,000,000 \\
\text { times }\end{array}$ \\
\hline $\begin{array}{c}\text { Read-write } \\
\text { speed }\end{array}$ & Fastest & Faster & Faster & Slow & General & Faster \\
\hline $\begin{array}{c}\text { Storage } \\
\text { characteristics }\end{array}$ & Volatile & Volatile & Non-volatile & Non-volatile & Non-volatile & Non-volatile \\
\hline Interface & $\begin{array}{c}\text { Parallel } \\
\text { port }\end{array}$ & $\begin{array}{c}\text { Parallel } \\
\text { port }\end{array}$ & $\begin{array}{c}\text { Serial / } \\
\text { parallel port }\end{array}$ & $\begin{array}{c}\text { Serial / } \\
\text { parallel port }\end{array}$ & $\begin{array}{c}\text { Serial / } \\
\text { parallel port }\end{array}$ & $\begin{array}{c}\text { Serial / } \\
\text { parallel port }\end{array}$ \\
\hline Price & $\begin{array}{c}\text { More } \\
\text { expensive }\end{array}$ & Cheap & Expensive & Cheap & Cheap & Moderate \\
\hline $\begin{array}{l}\text { Software } \\
\text { support }\end{array}$ & Simple & Complex & Simple & Simple & Simple & Complex \\
\hline Application & $\begin{array}{c}\text { Small } \\
\text { capacity } \\
\text { cache }\end{array}$ & Bulk cache & $\begin{array}{c}\text { Save data } \\
\text { fast and } \\
\text { power-down }\end{array}$ & $\begin{array}{c}\text { Small } \\
\text { amount of } \\
\text { data, } \\
\text { constant } \\
\text { storage }\end{array}$ & $\begin{array}{c}\text { Program, } \\
\text { data storage }\end{array}$ & $\begin{array}{c}\text { Large-capacity } \\
\text { data storage }\end{array}$ \\
\hline
\end{tabular}

Direct current (DC) regulated power supply circuit is divided into two parts: switch and linear DC regulated power supply. Linear DC voltage stabilizing power supply has small ripple, but low efficiency and high calorific value. While, switching DC voltage stabilizing power supply has wide range, small heat generation, and high efficiency, but its ripple is higher than linear DC voltage stabilizing power supply. Combining the advantages and disadvantages of the two power sources and circuit requirements, the two power sources are combined for power supply.

\subsection{Design of wireless vibration base station}

As the data transfer station of multiple monitoring nodes, the base station will affect the data storage and operation performance when monitoring data is larger in size. Therefore, the base station should have strong operation and storage capacity.

The base station can encounter the different operational problems and normally adopts wired mode to supply power to the high-energy base station.

The design scheme of wireless network base station is shown in figure 4 . The base station of wireless network includes control center, data storage, radio frequency transmission, Ethernet communication, and power supply.

Similarly, designing with modularity is beneficial for adding or removing different functional modules.

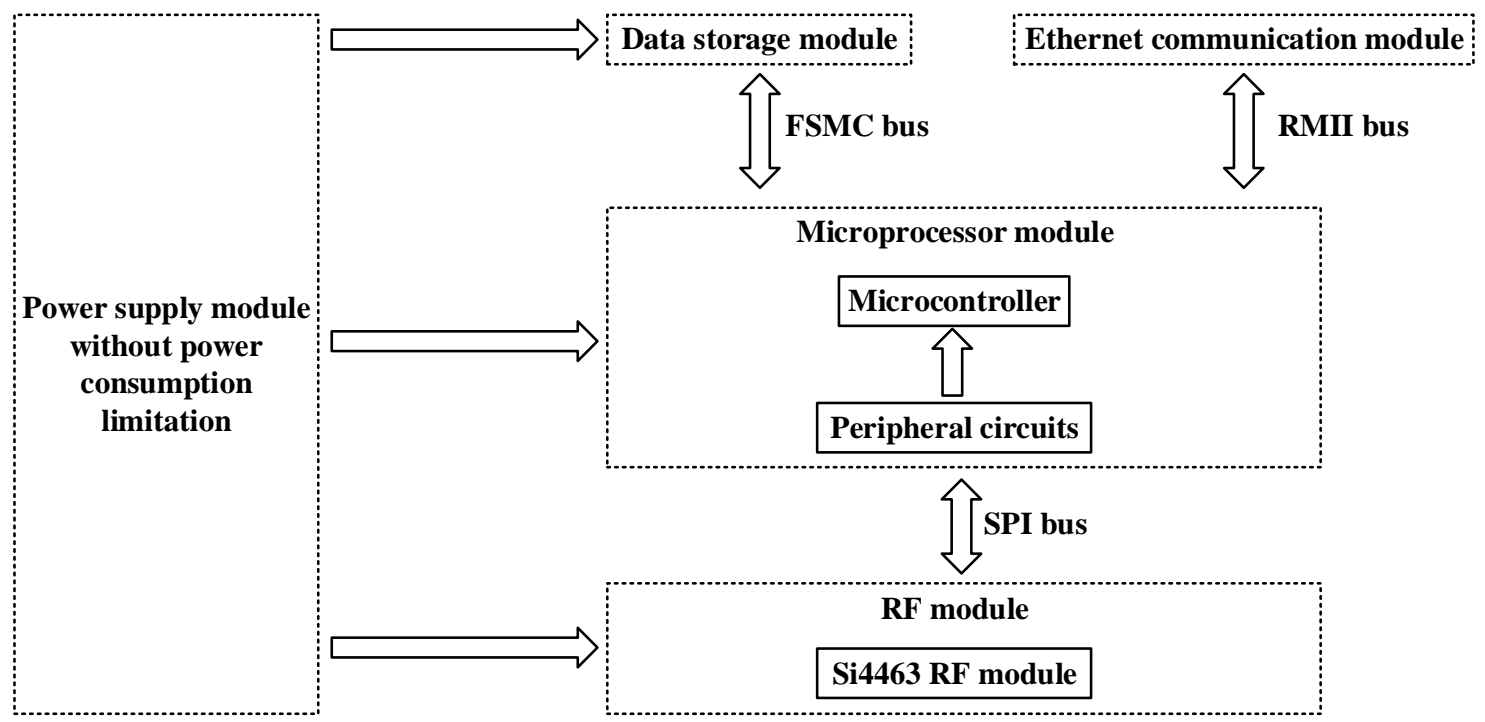

Figure 4: Design of a wireless network base station. 
Since the base station needs large memory SRAM and the processor with FSMC interface, the 32-bit microprocessor STM32F407 with ARM Cortex-M4 kernel is adopted in this study. Although the STM32F407 integrates an Ethernet MAC controller, it does not provide a physical layer interface. Therefore, in this study, DP83848 is used as the physical layer interface device, and RMII interface is used to connect STM32F407, which can simplify the circuit connection.

Generally, memory is divided into internal and external storages, as the name describes internal storage is kept inside the microprocessor. In the external memory, SRAM memory has fast read-write speed, high efficiency, and low energy consumption. It is not subject to the limit of erasing times, and does not need to refresh the circuit to meet the requirements of memory. In this study, CMOS static memory chip IS62WV102416 is used for the data storage unit. The chip has a capacity of $2 \mathrm{M}$. bytes, a total of 20 address lines and 16 data lines, and a read-write speed between 45-55 ns.

\subsection{Design of wireless network communication protocol}

The sensor nodes of wireless mechanical vibration monitoring network have weak computing capacity and the transmission protocol of wireless network is more simplified than traditional wireless network. Moreover, the reliable transmission of data is strong, so it is necessary to take the task characteristics as the design direction in the network communication protocol to meet the system requirements.

In wireless sensor network, network nodes are in a complex environment, most of them are in a static state, and the energy of network nodes is limited, so it is necessary to reduce energy consumption to extend the service life of sensor network. Therefore, it is necessary to provide a network topology for wireless sensor monitoring based on cost saving and efficiency improvement.

In the commonly used network topologies, the single-hop star network has a simple structure and low network delay, but the network coverage is small, and the network nodes are highly affected by the network throughput.

The multi-hop tree network structure has a wide coverage and flexible deployment of sensor nodes, which can also avoid interference from distance or environment. However, it has the disadvantages of high energy consumption and low transmission rate. In this study, two network structures are optimized for design, as shown in figure 5.

Star network structure is adopted to increase base station nodes, coverage area, and node flexibility. According to the principle of proximity, network nodes are grouped to reduce the number of nodes in each network, which not only considers the advantages of the above two network topologies, but also improves their defects.

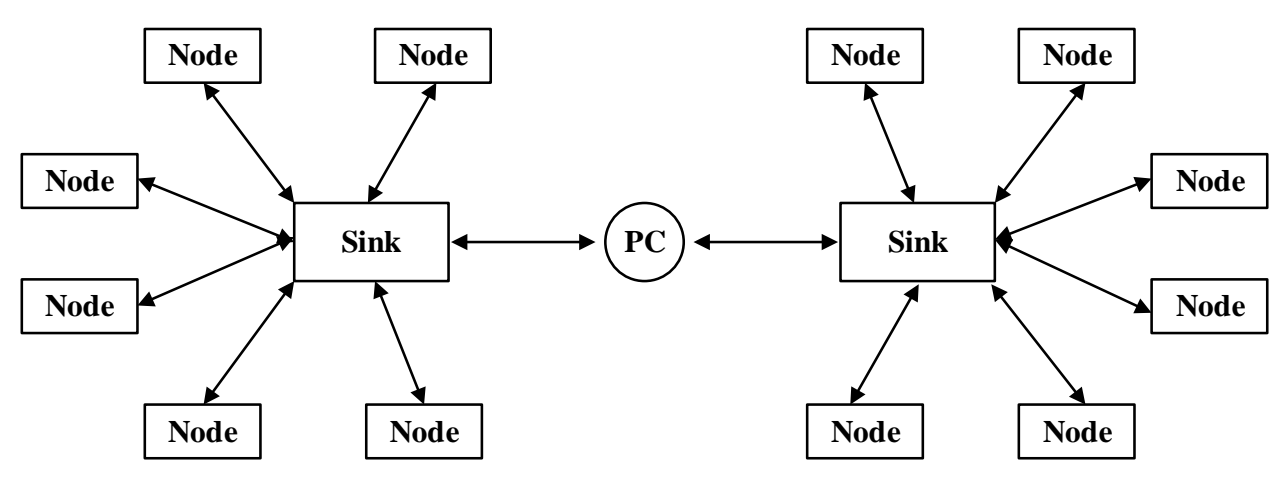

Figure 5: Network topology of multi-base station nodes.

In the wireless mechanical vibration monitoring network, the data collected by the monitoring node is transmitted to the base station with the wireless method, and then transmitted to the host computer through wire, so the network data packet format needs to be standardized. To meet the requirements of wireless network data transmission, four packet structures are required, which are: wireless transmission monitoring, command, request, and reply packets.
The monitoring node sends out the wireless transmission monitoring and request packet, and the base station receives it. The base station sends the wireless transmission reply and command packets, and the monitoring node receives them.

The definition of wireless transmission monitoring, command, and request (reply) packets is shown in table 4 . 
Table 4. Definition of data packets

\begin{tabular}{|c|c|c|c|}
\hline Length (Byte) & $\begin{array}{c}\text { Wireless transmission } \\
\text { monitoring packet }\end{array}$ & $\begin{array}{c}\text { Wireless transmission } \\
\text { command packet }\end{array}$ & $\begin{array}{c}\text { Wireless transmission } \\
\text { request (reply) packet }\end{array}$ \\
\hline Leader sequence & 8 & 8 & 8 \\
\hline $\begin{array}{c}\text { Synchronization } \\
\text { word }\end{array}$ & 1 & 1 & 1 \\
\hline Address & 1 & 1 & 2 \\
\hline Package information & 2 & 1 & 1 \\
\hline Control word & 1 & $\mathrm{X}$ & $\mathrm{X}$ \\
\hline Data & $\mathrm{X}$ & $\mathrm{X}$ & $\mathrm{X}$ \\
\hline CRC & $\mathrm{X}$ & & \\
\hline
\end{tabular}

In the wireless transmission monitoring packet, the leading sequence consists of "0101" or "1010", synchronizes the receiver and sender of the data, and notifies the receiver to prepare for receiving. The synchronization word is located after the preamble sequence, aligns the data during the transmission process, and informs the receiver that the data to be received is a valid data field, and the field length is generally 1 byte. The address represents that of the receiving packet node, the field length of the destination address is 1 byte, and the terminus address is " $0 \mathrm{x} 00$ ", " $0 \mathrm{xFF}$ " means that the packet is a broadcast packet. Packet information includes data flag bit and packet serial number. The data flag bit indicates whether the sent packet is the last packet, and the packet serial number represents the current packet serial number received, with a length of 2 bytes. The control byte is used to describe the nature of the monitoring data packet and is 1 byte in length. The data indicates that of the equipment status monitoring, and the CRC check is used to verify the correctness of the received data packet.
In a wireless transmission command packet, higher two bits of the control byte represent the packet category and the remaining bits represent the instruction opcode. The data indicates the parameters required to execute the command sent by the base station to the monitoring node. In the wireless transmission request (reply) packet, if the monitoring node sends the request packet, the data are all 0 . When the base station sends a reply packet, the data represents the network address assigned to the requesting node by the terminal.

\section{Results and Discussion 3.1 Reliable transmission test of data}

Figures 6 and 7 respectively show the length of the received data and the data loss rate when the data transmission length is 16389 after 10 data transmission experiments. As can be observed from the figure, the data loss rate is all lower than $0.2 \%$, indicating that the monitoring data meet necessities of mechanical vibration monitoring during transmission.

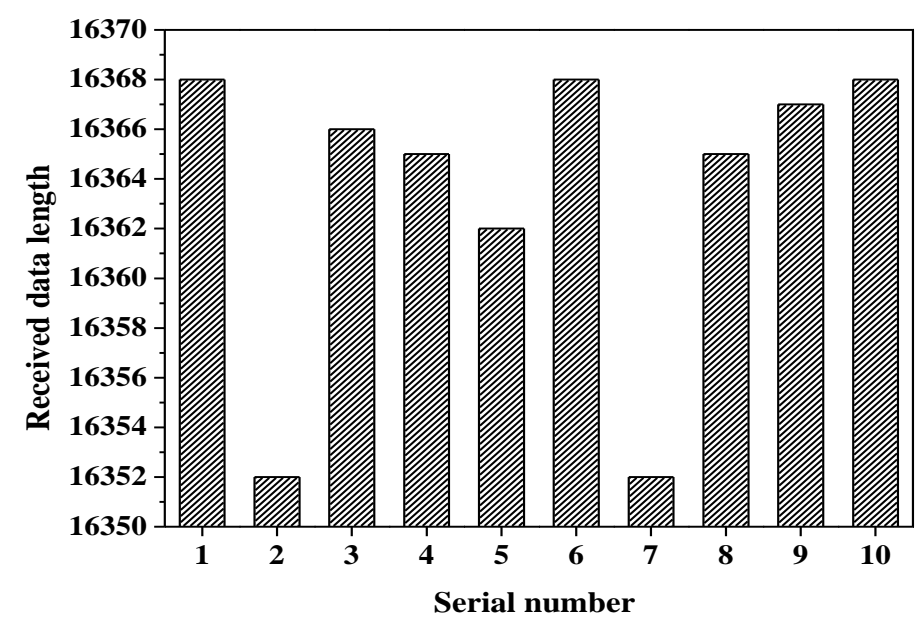

Figure 6: Received data length under transmission experiments 


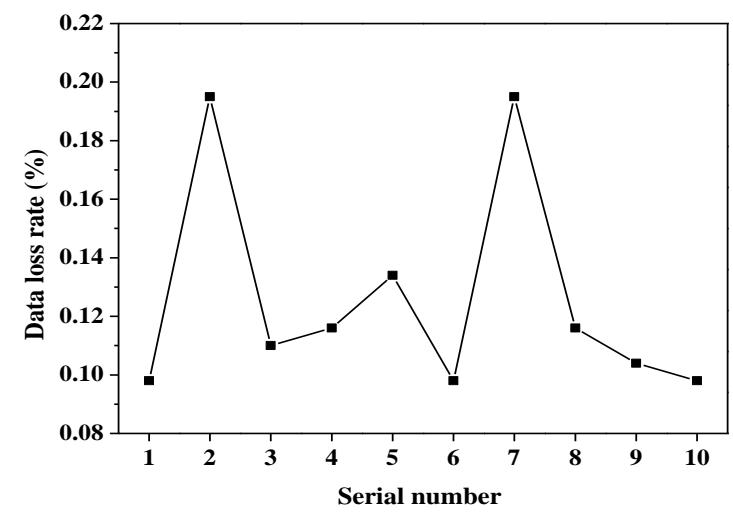

Figure 7: Data loss rate under transmission experiments

\subsection{Comparison of system performance}

To verify the effectiveness of the wireless monitoring node designed in this study, a Fourier transform spectrum comparison is performed with the data monitored by the YD-82 piezoelectric acceleration sensor. The comparison results of the main spectral lines are shown in figure 8 . The YD-82 sensor monitors the data through a wired method, with a sensitivity of $189.4 \mathrm{mV} / \mathrm{g}$, a frequency of 0.1 $2000 \mathrm{~Hz}$, having measurement range of $1000 \mathrm{~g}$, and an operating temperature of $-40-150{ }^{\circ} \mathrm{C}$ [14-15].
As can be observed from figure 8, the frequency and amplitude of the main spectral lines of wired monitoring and wireless monitoring are close to each other, but the spectral line amplitude of wireless monitoring is lower than that of wired monitoring. This is because the position deviation and angle deviation occurred during the installation of the sensor, resulting in certain errors in the measurement results. Therefore, the wireless network monitoring system designed in this study can meet the vibration monitoring requirements of mechanical equipment.

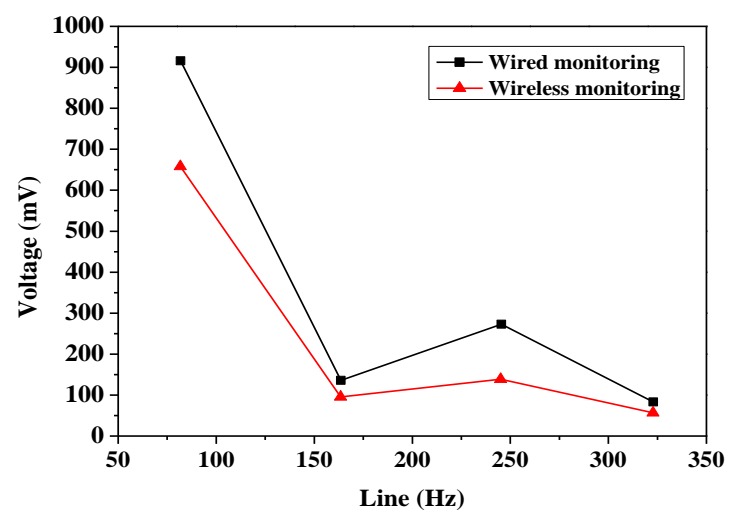

Figure 8: Comparison of the main spectral lines of wired monitoring and wireless monitoring.

\section{Conclusions}

In this research, to study the application of wireless network in remote monitoring of electromechanical equipment, the wireless vibration sensor monitoring system is designed. The wireless vibration monitoring node and the wireless vibration base station are modularized, the wireless network topology is improved, the packet format is standardized, the data transmission performance of the designed monitoring system is tested, and the performance of the designed monitoring system is compared with that of YD-82 piezoelectric acceleration sensor.
It is found that after 10 data transmission experiments, the data loss rate of the designed monitoring system is lower than $0.2 \%$, and the spectral line amplitude is lower than that of YD-82 sensor, which meet the remote monitoring requirements of electromechanical equipment.

However, the results of this study are still constrained by wireless short-range low-energy data transmission.

In the future research, the computing capacity and data compression of nodes will be improved to reduce the amount of wireless data transmission and achieve better monitoring performance. 


\section{Acknowledgements}

The study was supported by Science and Technology Projects in Shandong Province in 2016"Research on Private Information Security Model in Cloud Comput ing, China (plan No:J16LN58)".

\section{References}

[1] Rathnayaka S B, See K Y, Li K. Inductively coupled on-line impedance measurement for condition monitoring of electrical equipment. IET Science, Measurement \& Technology, 2018, 12(3), pp. 382-387.

[2] Agunov A V, Grishchenko A V, Kruchek V A, et al. A method of using neural fuzzy models to determine the technical state of a diesel locomotive's electrical equipment. Russian Electrical Engineering, 2017, 88(10), pp. 634638.

[3] Ichikawa N. Experimental consideration of the electrostatically induced voltage generated when a charged body approaches a metal box and moves away from the box. IEEE Transactions on Dielectrics and Electrical Insulation, 2017, 24(2), pp. 1203-1209.

[4] Dilip U Shenoy, Vinay Sharma, Shiva Prasad, H C (2020). Strategic Evaluation In Optimizing The Internal Supply Chain Using Topsis: Evidence In A Coil-Winding Machine Manufacturer. Acta Mechanica Malaysia, 3(1): 1-4, DOI: 10.26480/amm.01.2020.01.04

[5] Samijayani O N, Fauzi I, Zasyi M. Multi-Sensing Wireless Sensor Network for Smart Building System. Advanced Science Letters, 2017, 23(4), pp. 3660-3664.

[6] Lee Y, Kim J. Monitoring ON-Resistance of MOSFET Devices in Real Time for SVPWM-VSI With Direct Compensation. Canadian Journal of Electrical and Computer Engineering, 2018, 41(1), pp. 28-34.
[7] S. Sathishkumar, M. Kannan Structural (2020). Thermal and Thermo-Mechanical Analysis of Four Stroke Petrol Engine Piston Using Cae Tools. Acta Mechanica Malaysia, 3(1): 5-10

[8] Xue J R, Li B. Clinical application of electrical velocimetry in monitoring pediatric blood volume after cardiopulmonary bypass. Zhonghua er ke za zhi= Chinese journal of pediatrics, 2017, 55(12), pp. 932-936.

[9] Soh Hui Gian, Shahreen Kasim, Rohayanti Hassan, Zalmiyah Zakaria, Hairulnizam Mahdin, Azizul Azhar Ramli, Mohd Farhan Md Fudzee, Mohamad Aizi Salamat (2019). Online Activity Duration Management System for Manufacturing Company. Acta Electronica Malaysia, 3(2): 01-08.

[10] Samijayani O N, Fauzi I, Zasyi M. Multi-Sensing Wireless Sensor Network for Smart Building System. Advanced Science Letters, 2017, 23(4), pp. 3660-3664.

[11] Khan B M, Bilal R, Young R. Fuzzy-TOPSIS based cluster head selection in mobile wireless sensor networks. Journal of Electrical Systems and Information Technology, 2018, 5(3), pp. 928943.

[12] Hesham Enshasy, Qasem Abu Al-Haija, Hasan Al-Amri, Mohamed Al-Nashri, Sultan AlMuhaisen (2019). A Schematic Design of HHO Cell as Green Energy Storage. Acta Electronica Malaysia, 3(2): 09-15.

[13] Govindasamy J, Punniakody S. A comparative study of reactive, proactive and hybrid routing protocol in wireless sensor network under wormhole attack. Journal of Electrical Systems and Information Technology, 2018, 5(3), pp. 735-744.

[14] Dai K, Wang X, Yi F, et al. Triboelectric nanogenerators as self-powered acceleration sensor under high-g impact. Nano Energy, 2018, 45, pp. 84-93.

[15] Ihtisham ul Haq (2019). Analytical Approximate Solution Of Non-Linear Problem By Homotopy Perturbation Method (Hpm). Matrix Science Mathematic, 3(1): 20-24. 